\title{
Toward a Geometric Characterization of Intrinsic Ultracontractivity for Dirichlet Laplacians
}

\author{
PEdro J. MÉndeZ-HernándeZ
}

\section{Introduction}

Let $D$ be a domain in $\mathbb{R}^{2}$ and let $p_{t}^{D}(x, y)$ be the heat kernel of $e^{-t H}$, where $H$ is minus one half the Dirichlet Laplacian in $D$. We assume that $\varphi_{0}$, the eigenfunction of $H$ corresponding to the first eigenvalue $\lambda$, is positive - a mild assumption which holds for a very large class of domains, including all those considered in this paper. Because

$$
p_{t}^{D}(x, y) \leq \frac{1}{(2 t \pi)^{n / 2}} \exp \left\{\frac{-|x-y|^{2}}{2 t}\right\},
$$

the symmetric Markovian semigroup $e^{-t H}$ is ultracontractive; that is, it maps $L^{2}(D)$ into $L^{\infty}(D)$ for all $t>0$. Following [11], we say that $D$ is intrinsically ultracontractive (IU) if the symmetric Markovian semigroup $e^{-t \tilde{H}}$ in $L^{2}\left(D, \varphi_{0}^{2} d x\right)$ given by the kernel

$$
\tilde{p}_{t}^{D}(x, y)=\frac{e^{\lambda t} p_{t}^{D}(x, y)}{\varphi_{0}(x) \varphi_{0}(y)}
$$

is ultracontractive. This is equivalent (see [11]) to the existence of $a_{t}>0$ and $b_{t}>$ 0 depending only on $D$ and $t$ and such that, for all $x, y \in D$,

$$
b_{t} \varphi_{0}(x) \varphi_{0}(y) \leq p_{t}^{D}(x, y) \leq a_{t} \varphi_{0}(x) \varphi_{0}(y) .
$$

Because of its analytic and probabilistic consequences, intrinsic ultracontractivity has been widely studied by many authors (see e.g. [3; 4]). Sufficient conditions for IU can be found in $[2 ; 8 ; 11]$. The results in these papers do not give necessary and sufficient conditions. It seems to be very difficult (and perhaps impossible) to find a geometric characterization for IU without restricting to some subclasses of domains. However, there is a conjecture for a geometric characterization of IU for a certain class of simply connected domains. We will state it later in this section. The purpose of this paper is to provide some partial results related to this conjecture. Before we state the conjecture and our main results, we need to introduce some notation and present some definitions.

Received March 10, 1999. Revision received August 30, 1999.

Partially supported by the National Science Foundation and the Purdue Research Foundation. 
Definition 1. Let $D$ be a simply connected domain in the complex plane. We say that $D$ has the wide access property (WA) if there exists a fixed point $o \in D$ such that, for any $x \in D$, there is a path $\Gamma$ from $o$ to $x$ with

$$
\inf _{z \in \Gamma} d(z) \geq C_{\Gamma} d(x),
$$

where $C_{\Gamma}$ is a constant independent of $x$ and $d(y)$ denotes the distance from $y$ to the boundary of $D$. We will often call domains with this property WA domains.

REMARK 1. WA domains have also been referred to as $\Gamma$-domains (see $[4 ; 18]$ ).

Domains that are above the graph of an upper semicontinuous function give typical examples of domains with the wide access property. In fact, this case was studied by Bañuelos and Davis in [7], where a simple geometric characterization for IU is given. This was the only known geometric characterization of IU for a large class of domains with the wide access property. In this paper we give a necessary condition for IU in any simply connected domain in terms of areas of Whitney cubes. We also extend the Bañuelos-Davis result by proving that such geometrical condition is equivalent to IU for certain types of WA domains and that, in general WA domains, it implies that for all $x \in D$ there exist $a_{t}^{x}>0$ and $b_{t}^{x}>0$ such that

$$
a_{t}^{x} \varphi_{0}(x) \varphi_{0}(y) \leq p_{t}^{D}(x, y) \leq b_{t}^{x} \varphi_{0}(x) \varphi_{0}(y)
$$

for all $y \in D$. Domains with this property are often called "one-half intrinsic ultracontractive" because of the uniformity in one of the variables when the other is held fixed. This property was first studied in [6] where it was proved that it implies, among other things, the following asymptotic behavior of the heat kernel $p_{t}^{D}(x, y):$ Fix $x \in D$; then

$$
\lim _{t \rightarrow \infty} \frac{e^{\lambda t} p_{t}^{D}(x, y)}{\varphi_{0}(x) \varphi_{0}(y)}=1
$$

uniformly in $y$. One-half intrinsic ultracontractivity has many interesting probabilistic corollaries (see [6]). For instance, it implies that $D$ satisfies the "finite lifetime" condition defined in (9). We will briefly mention this connection below.

Following Stein [19], a Whitney cube decomposition $\mathcal{F}$ of $D$ is a collection $\mathcal{F}=$ $\left\{Q_{1}, Q_{2}, \ldots\right\}$, where $Q_{i}$ are cubes whose sides are parallel to the axes and

(1) $Q_{i}^{o} \cap Q_{j}^{o}=\emptyset$ for $i \neq j$;

(2) $\bigcup_{k} Q_{k}=D$;

(3) diam $Q_{k} \leq \operatorname{dist}\left(Q_{k}, D^{c}\right) \leq 4 \operatorname{diam} Q_{k}$ for all $k$.

Here (and in the rest of the paper), $\operatorname{dist}\left(Q_{k}, D^{c}\right)=\inf _{x \in Q_{k}} \operatorname{dist}\left(x, D^{c}\right)$ and $\operatorname{diam} Q_{k}$ is the diameter of $Q_{k}$. For $Q_{\alpha}, Q_{\beta} \in \mathcal{F}$, we say that $Q_{\alpha}=Q_{0} \rightarrow$ $Q_{1} \rightarrow \cdots \rightarrow Q_{n}=Q_{\beta}$ is a Whitney chain connecting $Q_{\alpha}$ to $Q_{\beta}$ of length $n$ if:

(i) $Q_{i} \in \mathcal{F}$ for $0 \leq i \leq n$; and

(ii) $Q_{i} \cap Q_{i+1} \neq \emptyset$ for $0 \leq i \leq n-1$.

We define the Whitney distance $\tilde{\rho}_{D}\left(Q_{\alpha}, Q_{\beta}\right)$ to be the length of the shortest Whitney chain connecting $Q_{\alpha}$ to $Q_{\beta}$. If $x, y \in D$ then we define $\tilde{\rho}_{D}(x, y)=$ $\tilde{\rho}_{D}\left(Q_{x}, Q_{y}\right)$, where $x \in Q_{x}$ and $y \in Q_{y}$. 
For $x, y \in D$, define the quasihyperbolic distance as

$$
\rho_{D}(x, y)=\inf _{\tilde{\gamma}} \int_{\tilde{\gamma}} \frac{1}{d(s)} d s,
$$

where the infimum is taken over all rectifiable curves $\tilde{\gamma}$ joining $x$ to $y$. A quasihyperbolic geodesic $\tilde{\gamma}_{x}^{y}$ is an arc for which this infimum is attained; see Martin [15]. It follows easily from the definition that there exist $b_{1}, b_{2}, b_{3}$ such that

$$
b_{1} \rho_{D}(x, y)-b_{2} \leq \tilde{\rho}_{D}(x, y) \leq b_{3} \rho_{D}(x, y)
$$

for all $x, y \in D$. Given $x \in D$, we set $\tilde{A}(x, \varepsilon)$ to be the area of the Whitney squares which intersect a quasihyperbolic geodesic from $o$ to $x$ and which have diameter less than $\varepsilon$.

Let

$$
\begin{aligned}
\tilde{A}(D, \varepsilon) & =\sup _{x \in D} \tilde{A}(x, \varepsilon), \\
\tilde{A}(D) & =\sup _{\varepsilon>0} \tilde{A}(D, \varepsilon) .
\end{aligned}
$$

The sufficient conditions for IU in WA domains given in [2; 8; 11] essentially provide upper bounds on $\tilde{A}(D, \varepsilon)$ to control its rate of convergence to 0 as $\varepsilon$ approaches 0 . The following conjecture, motivated by the results in [7], would, if true, extend and generalize all the aforementioned results.

Conjecture 1 (Bañuelos-Davis). If $D$ is a domain with the wide access property, then $D$ is IU if and only if $\tilde{A}(D, \varepsilon) \rightarrow 0$ as $\varepsilon \rightarrow 0$ and $\tilde{A}(D)<\infty$.

Our first theorem is a generalization of the necessary geometric condition for IU given in [7] to any simply connected domain.

TheOrem 1. Let $D$ be a simply connected domain. If $D$ is $I U$, then

$$
\lim _{\varepsilon \rightarrow 0} \tilde{A}(\varepsilon, D)=0 \quad \text { and } \quad \tilde{A}(D)<\infty .
$$

The following theorem asserts that in domains with the wide access property, (6) implies the weaker version of IU mentioned before.

THEOREM 2. Let D be a domain with the wide access property. If D satisfies (6), then for all $x \in D$ there exist $a_{t}^{x}, b_{t}^{x}>0$ such that

$$
a_{t}^{x} e^{-\lambda t} \varphi_{0}(x) \varphi_{0}(y) \leq p_{t}^{D}(x, y) \leq b_{t}^{x} e^{-\lambda t} \varphi_{0}(x) \varphi_{0}(y)
$$

for all $y \in D$.

In the proof of Theorem 2 we follow the arguments in [6], where Bañuelos and Davis prove Theorem 2 for any bounded domain. It turns out to be easy to prove Conjecture 1 for bounded domains with the wide access property. However, we must remark that the domains considered in Theorem 2 could have infinite area; therefore, Theorem 2 extends the results in [6] for these domains. 
As mentioned earlier, inequality (7) has some interesting probabilistic corollaries. Let $B_{t}$ be a two-dimensional Brownian motion and let $\tau_{D}$ be the first exit time from $D$. The heat kernel $p_{t}^{D}(x, y)$ gives the transition densities of $B_{t}$ killed on the boundary of $D$. If $h$ is a positive superharmonic function in $D$, the Doob $h$-conditioned Brownian motion is the Markov process with transition densities

$$
p_{D}^{h}(t, x, y)=\frac{p_{t}^{D}(x, y) h(x)}{h(y)} .
$$

Then $P_{h}^{x}\left(\tau_{D}>t\right)=\int_{D} p_{D}^{h}(t, x, y) d y$ is the probability that the Doob $h$-process started at $x$ does not leave $D$ before time $t$. We will write $E_{x}^{h}$ for the mean associated with $P_{x}^{h}$. In the case of $h(\cdot)=G_{D}(\cdot, y)$, we denote $P_{x}^{h}$ and $E_{x}^{h}$ by $P_{x}^{y}$ and $E_{x}^{y}$, respectively. This is the Brownian motion started at $x$ and conditioned to terminate at $y$ without leaving the domain $D$. Using Theorem 2 and the arguments given in [6], we obtain the following result.

COROLlary 1. Let $\mathcal{H}$ be the set of all positive superharmonic functions in D. If $D$ satisfies the hypothesis of Theorem 2 and $h \in \mathcal{H}$, then

(1) $\lim _{t \rightarrow \infty} \frac{e^{\lambda t} p_{t}^{D}(x, y)}{\varphi_{0}(x) \varphi_{0}(y)}=1$ uniformly in $y$ for each $x$.

(2) $\int_{D} \varphi_{0}(y) h(y) d y<\infty$.

(3) $\lim _{t \rightarrow \infty} e^{\lambda t} P_{h}^{x}\left(\tau_{D}>t\right)=\frac{\varphi_{0}(x)}{h(x)} \int_{D} h(y) \varphi_{0}(y) d y$.

(4) $\sup _{x \in D, t>0, h \in \mathcal{H}} e^{\lambda t} P_{h}^{x}\left(\tau_{D}>t\right)<\infty$.

A domain is said to satisfy the finite lifetime condition if

$$
\sup _{h \in \mathcal{H}, x \in D} E_{h}^{x}\left(\tau_{D}\right)<\infty .
$$

Since $E_{h}^{x}\left(\tau_{D}\right)=\int_{0}^{\infty} P_{h}^{x}\left(\tau_{D}>t\right) d t$, it is clear that Corollary 1(4) implies (9). Thus the results in this paper generalize Theorem 1 in [18], which gives a geometrical characterization for finite lifetime in domains with the wide access property.

There are basically two general (and very distinct) methods available to prove sufficient conditions for IU. The first is to use the following probabilistic characterization. For each $t>0$, there exists a compact set $K_{t}$ such that, for all $x \in D$,

$$
P^{x}\left(B_{t} \in K_{t} \mid \tau_{D}>t\right)>a_{t},
$$

where $a_{t}$ is independent of $x$. Using this characterization and the methods in [8] we prove the conjecture for WA domains given by tubes along geodesics. More precisely, let $D$ be a domain with the wide access property, and define:

(1) $\rho^{*}(r)=\sup _{x \in D_{r}^{c}} \tilde{\rho}_{D}(o, x)$, where $D_{r}=\left\{x \in D: d(x)>1 / 2^{r}\right\}$.

(2) $\rho_{\tilde{\gamma}}(r)=\sup _{x \in D_{r}^{c} \cap \tilde{\gamma}} \tilde{\rho}_{D}(o, x)$, where $\tilde{\gamma}$ is a quasihyperbolic geodesic. 
We say that $D$ is a tube along a geodesic $\tilde{\gamma}$ if there exist a quasihyperbolic geodesic $\tilde{\gamma}$ and positive integers $n_{D}, m_{D}$ such that

$$
\rho^{*}(r) \leq \rho_{\tilde{\gamma}}\left(r+n_{D}\right)+m_{D} .
$$

For these domains, (6) is equivalent to $\tilde{A}(D)<\infty$. If $D$ is given by the graph of a function, Bañuelos and Davis proved that $D$ is IU if and only if $\tilde{A}(D)<\infty$. Geometrically, the Bañuelos-Davis result is the case of $\tilde{\gamma}$ being a line. However, their argument does not seem to work for twisted tubes. The following theorem extends their result to twisted tubes.

THEOREM 3. Let D be a tube along the geodesic $\tilde{\gamma}$. Then $D$ is IU if and only if $\tilde{A}(D)<\infty$.

If $D$ is a domain with the wide access property such that $\tilde{A}(D)<\infty$, it is easy to prove that $D$ is a countable union (could also be finite) of tubes along geodesics; thus, Theorem 3 seems to be a necessary step toward the solution of the conjecture. In fact, the argument used to prove Theorem 3 proves Conjecture 1 when $D$ is the union of a finite number of tubes. However, up to this point we have not been able to make the argument work for the general case even under the assumption that $\tilde{A}(D, \varepsilon) \rightarrow 0$ as $\varepsilon \rightarrow 0$.

The second method, used in $[2 ; 11]$ and described in Section 6 , is based on the theory of logarithmic Sobolev inequalities. This is a powerful analytic technique which, when it works, gives information about $e^{-t H}$ for a wider class of Schrödinger operators. In addition, it provides detailed information on the behavior of the constants $a_{t}$ and $b_{t}$ as functions of $t$. Following this approach, we prove the conjecture for a special case of tubes along geodesics and for Schrödinger operators. More precisely, let $D$ be a tube along $\tilde{\gamma}$ and let $n_{k}$ be the number of Whitney cubes of diameter $\sqrt{2} / 2^{k}$ that intersect $\tilde{\gamma}$. (In [19] it is proved that we can take a Whitney decomposition $\mathcal{F}$ such that, for any $Q \in \mathcal{F}$, diam $Q=\sqrt{2} / 2^{k}$ for some $k \in Z$.) Consider the operator $H=H_{0}+V$, where $H_{0}$ is a second-order divergence form uniformly elliptic operator with bounded measurable coefficients and with Dirichlet boundary conditions and where $V$ is a potential in the Kato class $\mathbf{K}_{2}$. For more information on these operators, see Davies [11]. We define $e^{-t \tilde{H}}$ and the notion of intrinsic ultracontractivity of $e^{-t \tilde{H}}$ in the same way we did for the Dirichlet Laplacian. By studying the behavior of $d(x)^{2} \rho_{D}(o, x)$ and using the arguments in [2] and [11], we obtain the following result.

TheOREM 4. Let $D$ be a tube along $\tilde{\gamma}$. If $\tilde{A}(D)<\infty$ and

$$
n_{k+1} \leq 3\left(n_{1}+\cdots+n_{k}\right)
$$

for $k$ large enough, then $e^{-t H}$ is intrinsically ultracontractive.

We must remark that the arguments in the proof of Theorem 3 imply Theorem 4 just when $V=0$ and $H_{0}$ is the Dirichlet Laplacian. As a matter of fact, Theorem 4 seems to be new even for the case of tubes given by the graph of a function with $V=0$ and $H_{0}$ a uniformly elliptic operator. Following this approach, we can 
prove the conjecture for bounded WA domains and also prove that, in a general WA domain, (6) implies that $D$ is intrinsically supercontractive. In other words, the operators $e^{-t \tilde{H}}$ are bounded from $L^{p}\left(D, \varphi_{0}^{2}\right)$ to $L^{r}\left(D, \varphi_{0}^{2}\right)$ for all $t>0$ and $1<p \leq r<\infty$.

The paper is organized as follows. In Section 2 we set up some more notation and give some lemmas on the geometry of quasihyperbolic and hyperbolic geodesics. In Section 3 we prove Theorem 1, followed by the proof of Theorem 2 and Corollary 1 in Section 4. Theorem 3 is proved in Section 5, and in Section 6 we prove Theorem 4.

Throughout the paper, the letters $c, C$, will be used to denote constants which may change from line to line but which do not depend on the variables $x, y, z, \ldots$.

\section{Preliminaries}

Let $D$ be a simply connected domain in $\mathbb{R}^{2}$ with $z \in D$, and define $\sigma_{D}(z)=$ $1 /\left|F^{\prime}(0)\right|$, where $F$ is a conformal mapping sending the unit disc $B$ onto $D$ with $F(0)=z$. Let $x, y \in D$ define the hyperbolic distance between $x, y$ by

$$
d_{D}(x, y)=\inf _{\gamma} \int_{\gamma} \sigma_{D}(s) d s,
$$

where the infimum is taken over all rectifiable curves $\gamma$ from $x$ to $y$. A hyperbolic geodesic is an $\operatorname{arc} \gamma_{x}^{y}$ for which the infimum is attained. It can be shown that there exists a conformal map $\Phi_{x y}: B \rightarrow D$ with $\Phi_{x y}(0)=x, \Phi_{x y}(r)=y$ for some $r \in$ $(0,1)$, and $\gamma_{x}^{y}=\Phi_{x y}([0, r])$; see [16]. Define $\Gamma_{x}^{y}=\Phi_{x y}((-1,1))$.

Koebe's one-quarter theorem implies

$$
d_{D}(z) \leq\left|F^{\prime}(0)\right| \leq 4 d_{D}(z),
$$

and therefore

$$
d_{D}(x, y) \leq \rho_{D}(x, y) \leq 4 d_{D}(x, y) .
$$

If $D$ is a WA domain, Lemma 1 in [18] states that, for every $x \in D$, we can take $\Gamma$ to be either the hyperbolic geodesic $\gamma_{o}^{y}$ or the quasihyperbolic geodesic $\tilde{\gamma}_{o}^{y}$. Using this, the fact that $d_{D}(\cdot, \cdot)$ and $\rho_{D}(\cdot, \cdot)$ are equivalent metrics in any simply connected domain, and the Gromov theory of hyperbolic groups, Smits [17] proved the following lemma.

Lemma 1. Let $D$ be a simply connected domain. Then there exists a universal constant $K \geq 0$ such that, for all $x, y \in D$,

(i) $d_{D}\left(z_{1}, \tilde{\gamma}_{x}^{y}\right) \leq K$ for all $z_{1} \in \gamma_{x}^{y}$;

(ii) $d_{D}\left(z_{2}, \gamma_{x}^{y}\right) \leq K$ for all $z_{2} \in \tilde{\gamma}_{x}^{y}$.

Here and in the sequel we denote quasihyperbolic geodesics from $x$ to $y$ by $\tilde{\gamma}_{x}^{y}$ and hyperbolic geodesics by $\gamma_{x}^{y}$. Consequently, we define $A(D, \varepsilon)$ and $A(D)$ in the same way we define $\tilde{A}(D, \varepsilon)$ and $\tilde{A}(D)$ but taking Whitney cubes that intersect hyperbolic geodesics. In [18] Smits proved that, for any simply connected domain, $A(D)<\infty$ if and only if $\tilde{A}(D)<\infty$. We follow his argument to prove 
the following lemma, which tells us that we can use hyperbolic geodesics instead of quasihyperbolic geodesics in our results.

LeMma 2. If $D$ is a simply connected domain, then $\lim _{\varepsilon \rightarrow 0} A(D, \varepsilon)=0$ if and only if $\lim _{\varepsilon \rightarrow 0} \tilde{A}(D, \varepsilon)=0$.

Proof. Let $x \in D$ and $Q \in \mathcal{F}$, with $Q \cap \gamma_{o}^{x} \neq \emptyset$. Using Lemma 1, (11), and (5), we find a universal constant $K_{1}$ with

$$
\rho_{D}\left(Q, \tilde{\gamma}_{o}^{x}\right) \leq K_{1} .
$$

Thus there is $Q^{\prime} \in \mathcal{F}$ with $Q^{\prime} \cap \tilde{\gamma}_{o}^{x} \neq \emptyset$ and $\rho_{D}\left(Q, Q^{\prime}\right) \leq K_{1}$. Since the diameters of adjacent Whitney cubes differ at most by a factor of 4 , we have

$$
|Q| \leq 4^{2 K_{1}}\left|Q^{\prime}\right|
$$

where $|Q|$ denotes the Lebesgue measure (area) of $Q$. By the triangle inequality, for each Whitney cube $Q^{\prime}$ that intersects $\tilde{\gamma}_{o}^{x}$, there are at most $2 K_{1}+1$ cubes $\bar{Q}$ that intersect $\gamma_{o}^{x}$ with $\rho_{D}\left(Q^{\prime}, \bar{Q}\right) \leq K_{1}$. Hence

$$
A(\varepsilon, x) \leq 4^{2 K_{1}}\left(2 K_{1}+1\right) \tilde{A}\left(4^{K_{1}} \varepsilon, x\right)
$$

and the result follows by the symmetry of the argument.

We now describe some relationships between the hyperbolic distance and the Green function in $D$. It is well known that, for all $z \in B$,

$$
G_{B}(0, z)=\frac{1}{2 \pi} \log \frac{1}{|z|} \quad \text { and } \quad d_{B}(0, z)=\frac{1}{2} \log \frac{1+|z|}{1-|z|} .
$$

These identities are discussed in Hayman [14]. Solving for $|z|$ in terms of $d_{B}(0, z)$, one finds that

$$
G_{B}(0, z)=\frac{1}{2 \pi} \log \left(\operatorname{coth} e^{-2 d_{B}(0, z)}\right) \quad \text { for all } z \in B .
$$

Let $\Phi$ be a conformal map from $B$ to $D$, with $\Phi(0)=x$ and $\Phi(z)=y$. By the conformal invariance of both the Green function and the hyperbolic metric, we have

$$
G_{D}(y, x)=\frac{1}{2 \pi} \log \left(\operatorname{coth} e^{-2 d_{D}(x, y)}\right) \quad \text { for all } x, y \in D .
$$

Then there exist universal constants $a_{1}, a_{2}>0$ such that:

(i) $e^{-2 d_{D}(x, y)} \leq a_{1} G_{D}(x, y)$ for all $x, y \in D$;

(ii) $G_{D}(x, y) \leq a_{2} e^{-2 d_{D}(x, y)}$ provided that $d_{D}(x, y) \geq 1$.

Hence, for $x, y, w$ in $D$ with $d_{D}(y, w) \geq 1$ and $d_{D}(x, y) \geq 1$, we obtain

$$
\frac{G_{D}(x, y) G_{D}(y, w)}{G_{D}(x, w)} \leq a_{2}^{2} a_{1} e^{-2\left(d_{D}(x, y)+d_{D}(y, w)-d_{D}(x, w)\right)} .
$$

By [9, Prop. (1.3)] (note that, when Coornaert and Papadopoulus refer to the hyperbolic space, they endow $B$ with the metric $\left.\frac{1}{2} d_{D}(\cdot, \cdot)\right)$, there exists $a_{3}$ such that, for all $x, y \in D$, 


$$
d_{D}(x, y)+d_{D}(w, y)-d_{D}(x, w) \geq d_{D}\left(y, \gamma_{w}^{x}\right)-a_{3} .
$$

Thus, there exists a universal constant $a_{4}$ such that

$$
\frac{G_{D}(x, y) G_{D}(y, w)}{G_{D}(x, w)} \leq a_{4} e^{-2 d_{D}\left(y, \gamma_{w}^{x}\right)},
$$

provided that $d_{D}(x, y) \geq 1$ and $d_{D}(y, w) \geq 1$.

For the rest of this paper, $Z_{t}(x, y)$ denotes the $h$-conditioned Brownian motion starting at $x$, where $h(\cdot)=G_{D}(\cdot, y)$. The variance and covariance associated with the distribution of $Z_{t}(x, y)$ will be denoted by $\operatorname{var}_{x}^{y}$ and $\operatorname{cov}_{x}^{y}$. For any planar Borel set $O$, we denote by $P_{x}^{y}(O)$ the probability that $Z_{t}(x, y)$ ever hits $O$. The following results from Davis [12] and Bañuelos and Carroll [5] are fundamental in the proof of Theorem 1. (Recall that $|Q|$ denotes the area of the cube $Q$.)

THEOREM 5 [12]. There exists a constant $c_{1}$ such that, if $Q$ is a Whitney cube and if one of o or $y$ is a distance at least $\frac{1}{2} \operatorname{diam} Q$ from $Q$, then

$$
E_{o}^{y}\left(T_{Q}\right) \geq c_{1} P_{o}^{y}(Q)|Q|,
$$

where $T_{Q}=\int_{0}^{\tau_{D}} 1_{\left\{Z_{t} \in Q\right\}} d t$.

TheOREm 6 [12]. There exists a constant $c_{2}$ such that, if $Q$ and $R$ are Whitney cubes, then

$$
\left|\operatorname{cov}_{o}^{y}\left(T_{Q}, T_{R}\right)\right| \leq e^{-c_{2} \tilde{\rho}_{D}(Q, R)}|Q \| R| .
$$

Lemma 3 [5]. There exist constants $c_{3}$ and $c_{4}$ such that, if $d_{D}(o, y) \geq 1$, then

$$
P_{o}^{y}(Q) \geq c_{3} e^{-c_{4} d_{D}\left(z_{Q}, \gamma_{o}^{y}\right)},
$$

where $z_{Q}$ is the center of $Q$.

We end this section by pointing out several interesting and useful geometric and analytic properties of domains with the wide access property. Suppose $D$ is a domain with the wide access property; then it is clear that, for all $x \in D$,

$$
d(x) \leq \frac{1}{C_{\Gamma}} d(o) .
$$

We can therefore suppose that $\sup _{x \in D} d(x) \leq 1$. Furthermore, if $Q \in \mathcal{F}$ with $Q \cap \tilde{\gamma}_{o}^{x} \neq \emptyset$ and $z_{Q} \in \tilde{\gamma}_{o}^{x} \cap Q$, then

$$
\operatorname{diam} Q \geq \frac{1}{8 \sqrt{2}} d\left(z_{Q}\right) \geq \frac{C_{\Gamma}}{8 \sqrt{2}} d(x) .
$$

Thus, if $n_{k}$ is the number of Whitney cubes of diameter $\sqrt{2} / 2^{k}$ that intersect $\tilde{\gamma}_{o}^{x}$, then there exists $k_{x} \in N$ such that $n_{k}=0$ for $k \geq k_{x}$ and

$$
\tilde{A}(x, D)=\sum_{0}^{k_{x}} \frac{n_{k}}{2^{2 k}} \geq c d(x)^{2} \tilde{\rho}_{D}(o, x) .
$$

Since $\tilde{\rho}_{D}(o, x) \rightarrow \infty$ as $x \rightarrow \infty$, we conclude that $A(D)<\infty$ implies $d(x) \rightarrow$ 0 as $x \rightarrow \infty$, and by [10, Thm. 1.6.8] the spectrum of $H$ is discrete. 
Suppose now that $D$ satisfies (6) and that diam $Q_{x}=\sqrt{2} / 2^{m}$. From (14) there exists a $c$ such that

$$
\begin{aligned}
d(x)^{2} \rho_{D}(o, x) & =c d(x)^{2} \sum_{k=0}^{k_{x}} n_{k} \\
& \leq c \sum_{0 \leq k \leq m / 2} \frac{n_{k}}{2^{2 k}} \frac{1}{2^{2 m-2 k}}+c \sum_{m / 2 \leq k \leq k_{x}} \frac{n_{k}}{2^{2 k}} \\
& \leq c d(x) \sum_{0 \leq k \leq m / 2} \frac{n_{k}}{2^{2 k}}+c \sum_{m / 2 \leq k \leq k_{x}} \frac{n_{k}}{2^{2 k}} \\
& \leq c d(x) \tilde{A}(D)+c \tilde{A}\left(D, \frac{\sqrt{2}}{2^{m / 2}}\right) .
\end{aligned}
$$

Therefore, $d(x)^{2} \rho_{D}(o, x) \rightarrow 0$ as $d(x) \rightarrow 0$ and, by [7, Thm. 5] we conclude that $D$ is intrinsically supercontractive. That is, $e^{-t \tilde{H}}$ is bounded from $L^{p}(D)$ to $L^{r}(D)$ for all $t>0$ and for $1<p \leq r<\infty$. In a similar way we can prove that if $D$ is a bounded WA domain then $\rho_{D}(x) \leq c / d(x)$ and, by [7, Thm. 6], $D$ is IU.

\section{Proof of Theorem 1}

To prove Theorem 1, let us suppose that (6) is false. Using the results from [12] and [5] stated at the end of the previous section, we show that this implies the existence of sequences $\left\{z_{n}\right\}_{0}^{\infty} \subset D$ and $\left\{R_{n}\right\}_{0}^{\infty}$ (where $R_{n}$ is a union of Whitney cubes) such that, for some $\varepsilon>0$,

$$
\lim _{n \rightarrow \infty} P_{o}^{z_{n}}\left(T_{R_{n}}>\varepsilon\right)=1
$$

where

$$
T_{O}=\int_{0}^{\tau_{D}} 1_{\left\{Z_{t} \in O\right\}} d t
$$

is the time spent by $Z_{t}$ in a Borel set $O$ contained in $D$. Then we note that (17) contradicts the following consequence of intrinsic ultracontractivity (inequality (2.11) in [7]): there exist $c_{t}$ independent of $x$ and $y$ such that

$$
P_{x}^{y}\left(t<\tau_{D}<2 t\right) \geq c_{t} P_{x}^{y}\left(\tau_{D}>2 t\right) .
$$

Since IU implies (as mentioned before; see [2]) that

$$
\sup _{h \in \mathcal{H}, x \in D} E_{h}^{x}\left(\tau_{D}\right)<\infty,
$$

it follows by Theorem 1 in [18] that

$$
\tilde{A}(D)<\infty .
$$

We should remark that, although Theorem 1 of Smits [18] asserts that the finite lifetime condition is equivalent to $\tilde{A}(D)<\infty$ for any WA domain $D$, his proof of the necessary condition for finite lifetime is valid for any simply connected domain. 
Next, suppose that $\lim _{\varepsilon \rightarrow 0} \tilde{A}(D, \varepsilon) \neq 0$. Then there exist $\delta>0$ and sequences $\left\{z_{n}\right\}_{0}^{\infty},\left\{m_{n}\right\}_{0}^{\infty}$ (with $\lim _{n \rightarrow \infty} m_{n}=0$ ) such that

$$
\tilde{A}\left(z_{n}, \sqrt{2} / m_{n}\right) \geq \delta \text {. }
$$

Let $Q_{1}^{n}, \ldots, Q_{k_{n}}^{n}$ be the Whitney cubes that intersect $\tilde{\gamma}_{o}^{z_{n}}$ with diameter less than $\sqrt{2} / m_{n}$, and consider $T_{R_{n}}$ where $R_{n}=\bigcup_{i=1}^{k_{n}} Q_{i}^{n}$. For $n$ large enough we have

$$
\min \left\{d_{D}\left(o, Q_{i}^{n}\right), \tilde{\rho}_{D}\left(o, Q_{i}^{n}\right)\right\} \geq 1
$$

for $1 \leq i \leq k_{n}$; by Theorem 5 ,

$$
E_{o}^{z_{n}}\left(T_{R_{n}}\right) \geq \frac{c}{2} \sum_{i=0}^{k_{n}} P_{o}^{z_{n}}\left(Q_{i}^{n}\right)\left|Q_{i}^{n}\right|,
$$

(recall that $P_{o}^{z_{n}}\left(Q_{i}^{n}\right)$ denotes the probability that $Z_{t}\left(o, z_{n}\right)$ ever hits $\left.Q_{i}^{n}\right)$. Lemma 3 gives $c_{3}, c_{4}$ with

$$
P_{o}^{z_{n}}\left(Q_{i}^{n}\right) \geq c_{3} \exp \left\{-c_{4} d_{D}\left(z_{Q_{i}^{n}}, \gamma_{o}^{z_{n}}\right)\right\},
$$

where $z_{Q_{i}^{n}}$ is the center of $Q_{i}^{n}$.

Given that $Q_{i}^{n} \cap \tilde{\gamma}_{o}^{z_{n}} \neq \emptyset$, one sees from Lemma 1 that there exists a constant $c_{5}$ with

$$
E_{o}^{z_{n}}\left(T_{R_{n}}\right) \geq c_{5}\left|R_{n}\right| \geq c_{5} \delta
$$

Without loss of generality we can suppose that $\tilde{\rho}_{D}\left(Q_{i}^{n}, Q_{j}^{n}\right) \geq|i-j|$. Now, from Theorem 6 we obtain

$$
\begin{aligned}
\operatorname{var}_{o}^{z_{n}}\left(T_{R_{n}}\right) & \leq \sum_{i=1}^{k_{n}} \sum_{j=1}^{k_{n}}\left|\operatorname{cov}_{o}^{z_{n}}\left(T_{Q_{j}^{n}}, T_{Q_{i}^{n}}\right)\right| \\
& \leq c \sum_{i=1}^{k_{n}} \sum_{j=1}^{k_{n}} e^{-c_{2} \tilde{\rho}_{D}\left(Q_{i}^{n}, Q_{j}^{n}\right)}\left|Q_{j}^{n}\right|\left|Q_{i}^{n}\right| \\
& \leq c \sum_{i=1}^{k_{n}}\left|Q_{i}^{n}\right|^{2}+c \sum_{i=1}^{k_{n}} \sum_{j=i+1}^{k_{n}} e^{-c_{2}|i-j|}\left|Q_{j}^{n}\right|\left|Q_{i}^{n}\right| \\
& \leq \frac{c}{m_{n}^{2}}\left[\tilde{A}(D)+\sum_{i=1}^{k_{n}}\left|Q_{i}^{n}\right| \sum_{j=1}^{\infty} e^{-c_{2} j}\right] \\
& \leq \frac{c}{m_{n}^{2}} \tilde{A}(D) .
\end{aligned}
$$

Take $\varepsilon<c_{5} \delta / 2$; then (19) yields

$$
\begin{aligned}
P_{o}^{z_{n}}\left(T_{R_{n}} \leq \varepsilon\right) & \leq P_{o}^{z_{n}}\left(T_{R_{n}} \leq E\left(T_{R_{n}}\right)-\varepsilon\right) \\
& \leq P_{o}^{z_{n}}\left(\left|T_{R_{n}}-E\left(T_{R}\right)\right| \leq \varepsilon\right) \\
& \leq \frac{1}{\varepsilon^{2}} \operatorname{var}_{o}^{z_{n}}\left(T_{R_{n}}\right) \\
& \leq \frac{1}{\varepsilon^{2}} \frac{c}{m_{n}^{2}} \tilde{A}(D) .
\end{aligned}
$$


Thus $\lim _{n \rightarrow \infty} P_{o}^{z_{n}}\left(T_{R_{n}}>\varepsilon\right)=1$, and so we have

$$
\lim _{n \rightarrow \infty} P_{o}^{z_{n}}\left(\tau_{D}>\varepsilon\right)=1 .
$$

Taking $t=\varepsilon / 2$, one sees that

$$
\lim _{n \rightarrow \infty} P_{o}^{z_{n}}\left(\tau_{D}>2 t\right)=1 \text { and } \lim _{n \rightarrow \infty} P_{o}^{z_{n}}\left(2 t>\tau_{D}>t\right)=0 .
$$

However, this contradicts inequality (18) and so proves Theorem 1.

\section{Proof of Theorem 2}

Our proof follows the arguments in [6]. Let

$$
\begin{aligned}
D_{\beta} & =\{x \in D: d(x) \geq \beta\}, \\
F_{\beta} & =\left\{x \in D: x \in \gamma_{o}^{y}, y \in D_{\beta}\right\} .
\end{aligned}
$$

The following lemma follows from the arguments in [6, Sec. 3].

LEMma 4. Let $D$ be a domain in $\mathbb{R}^{2}$ that satisfies the finite lifetime condition and for which

$$
\lim _{\beta \rightarrow 0} \frac{1}{\varphi_{0}(y)} \int_{D \backslash F_{\beta}} G_{D \backslash F_{\beta}}(x, y) \varphi_{0}(x) d x=0
$$

uniformly in $y \in D$. Then, for each $x \in D$, there exist $a_{x}^{t}>0$ and $b_{x}^{t}>0$ depending only on $t$ and $x$ such that

$$
a_{x}^{t} e^{-\lambda t} \varphi_{0}(x) \varphi_{0}(y) \leq p_{t}^{D}(x, y) \leq b_{x}^{t} e^{-\lambda t} \varphi_{0}(x) \varphi_{0}(y) .
$$

By [18, Thm. 1], any WA domain $D$ such that $A(D)<\infty$ will satisfy the finite lifetime condition. Therefore, to prove Theorem 1 it is enough to show (20). Indeed, we prove a more general result as follows.

Lemma 5. Let D be a domain with the wide access property. If D satisfies (6), then

$$
\lim _{\beta \rightarrow 0} \sup _{h \in \mathcal{H}, y \in D} \frac{1}{h(y)} \int_{D \backslash F_{\beta}} h(x) G_{D \backslash F_{\beta}}(x, y) d x=0 .
$$

Proof. Let $h$ be in $\mathcal{H}$. Since $D$ is simply connected, the Riesz decomposition theorem implies the existence of two Borel measures $\mu_{1}, \mu_{2}$ such that $h(x)=$ $G_{D} \mu_{1}(x)+M_{D} \mu_{2}(x)$, where

$$
\begin{aligned}
G_{D} \mu_{1}(x) & =\int_{D} G_{D}(x, y) d \mu_{1}(y), \\
M_{D} \mu_{2}(x) & =\int_{\partial D} M_{D}(x, w) d \mu_{2}(w),
\end{aligned}
$$

and $M_{D}$ is the Martin kernel of $D$. Then, for any $y$ in $D$, 


$$
\begin{aligned}
& \frac{1}{h(y)} \int_{D \backslash F_{\beta}} G_{D \backslash F_{\beta}}(x, y) h(x) d x \\
& \leq \frac{1}{h(y)} \int_{D \backslash F_{\beta}} G_{D}(x, y) h(x) d x \\
& \leq \frac{1}{G_{D} \mu_{1}(y)} \int_{D} G_{D}(z, y) \int_{D \backslash F_{\beta}} \frac{G_{D}(y, x) G_{D}(x, z)}{G_{D}(z, y)} d x d \mu_{1}(z) \\
&+\frac{1}{M_{D} \mu_{2}(y)} \int_{\partial D} M_{D}(w, y) \int_{D \backslash F_{\beta}} \frac{G_{D}(y, x) M_{D}(x, w)}{M_{D}(w, y)} d x d \mu_{2}(w) .
\end{aligned}
$$

From the last inequality and the fact that

$$
\lim _{z \rightarrow w} \int_{D \backslash F_{\beta}} \frac{G_{D}(y, x) G_{D}(x, z)}{G_{D}(z, y)} d x=\int_{D \backslash F_{\beta}} \frac{G_{D}(y, x) M_{D}(x, w)}{M_{D}(w, y)} d x,
$$

one sees that it is enough to show that

$$
\lim _{\beta \rightarrow 0} \int_{D \backslash F_{\beta}} \frac{G_{D}(y, x) G_{D}(x, z)}{G_{D}(y, z)} d x=0
$$

uniformly in $y, z \in D$. That is, it is enough to prove (22) for the Brownian motion starting at $y$ and conditioned to end at $z$ without leaving $D$ uniformly in $y, z \in D$.

Fix $y, z \in D$, and consider

$$
\begin{aligned}
& A_{1}=\left\{x \in D \backslash F_{\beta}: d_{D}(x, y) \geq 1, d_{D}(x, z) \geq 1\right\}, \\
& A_{2}=\left\{x \in D \backslash F_{\beta}: d_{D}(x, y) \leq 1 \text { or } d_{D}(x, z) \leq 1\right\} .
\end{aligned}
$$

Clearly $D \backslash F_{\beta}=A_{1} \cup A_{2}$. We first deal with

$$
I_{A_{1}}=\int_{A_{1}} \frac{G_{D}(y, x) G_{D}(x, z)}{G_{D}(y, z)} d x .
$$

From inequality (13) one sees that

$$
I_{A_{1}} \leq c \int_{A_{1}} e^{-2 d_{D}\left(x, \gamma_{y}^{z}\right)} d x
$$

Recall that we can find a conformal map $\Phi_{y, z}: B \rightarrow D$ such that (a) $\gamma_{y}^{z}=$ $\Phi_{y, z}([0, r])$ for some positive $r$ in $[0,1)$ and (b) $\Gamma_{y}^{z}=\Phi_{y, z}[(-1,1)]$. It is clear that $d_{D}\left(x, \gamma_{y}^{z}\right) \geq d_{D}\left(x, \Gamma_{y}^{z}\right)$ and, by [18, Lemma 3],

$$
\begin{aligned}
e^{-2 d_{D}\left(x, \gamma_{y}^{z}\right)} & \leq e^{-2 d_{D}\left(x, \Gamma_{y}^{z}\right)} \\
& \leq 2\left(e^{-2 d_{D}\left(x, \Gamma_{o}^{z}\right)}+e^{-2 d_{D}\left(x, \Gamma_{o}^{y}\right)}\right) .
\end{aligned}
$$

Thus to prove that $I_{A_{1}}$ converges to zero uniformly in $y$ and $z$, is enough to show that

$$
\lim _{\beta \rightarrow 0} \int_{A_{1}} e^{-2 d_{D}\left(x, \Gamma_{o}^{y}\right)} d x=0
$$

uniformly in $y$. 
Let $\eta>0$ and take $k_{0}$ such that $A\left(\varepsilon / c_{\Gamma}, D\right)<\eta$ for $\varepsilon \leq \sqrt{2} / 2^{k_{0}}$. Let $Q_{1}, Q_{-1} \in$ $\mathcal{F}$ satisfy:

(i) $Q_{1} \cap \Phi_{o, y}[(0,1)] \neq \emptyset$ and $Q_{-1} \cap \Phi_{o, y}[(-1,0)] \neq \emptyset$;

(ii) $\operatorname{diam} Q_{1}=\operatorname{diam} Q_{-1}=\sqrt{2} / 2^{k_{0}}$.

Since $D$ is a WA domain, the pieces of geodesics that go from $Q_{1}$ to $Q_{-1}$ are contained in $D_{C_{\Gamma} / 2^{k}}$.

Following Definition 4.5 and Lemma 4.6 in [13], we can find a collection of Whitney cubes $\left\{\ldots, Q_{-2}, Q_{-1}, Q_{1}, Q_{2}, \ldots\right\}$ that satisfy the following conditions.

(1) For all $i \in N, \Phi_{o, y}[(0,1)] \cap Q_{i} \neq \emptyset$ and $\Phi_{o, y}[(-1,0)] \cap Q_{-i} \neq \emptyset$.

(2) For each $Q_{i}$, there exists a curve $I_{i}$ (often referred to as "cross cuts") that goes from a $w_{i}$ to $w_{i}^{\prime}$ with $w_{i}, w_{i}^{\prime} \in \partial D$ and such that: (a) $I_{i} \cap \Gamma_{o}^{y} \in Q_{i}$; (b) the pieces of curves that go from $w_{i}$ and $w_{i}^{\prime}$ to $Q_{i}$ are hyperbolic geodesics; and (c) length $\left(I_{i}\right) \leq \zeta$ diam $Q_{i}$, where $\zeta$ is a universal constant.

(3) Cube $Q_{i}$ is associated with an open set $\Omega_{i} \subset D$ such that $\Omega_{i} \cap \Omega_{j}=\emptyset$ for $i \neq j$. In addition, $D \cap \partial \Omega_{i}=I_{i+1} \cup I_{i}$ if $i>0$ and $D \cap \partial \Omega_{i}=I_{i} \cup I_{i-1}$ if $i<0$, with length $\left(\Gamma_{o}^{y} \cap \Omega_{i}\right)$ comparable to length $\left(I_{i}\right)$.

Define

$$
u_{o, y}= \begin{cases}1 & \text { if } x \in \Gamma_{o, y}, \\ \omega\left(x, D^{+}, \Gamma_{o, y}\right) & \text { if } x \in D^{+}, \\ \omega\left(x, D^{-}, \Gamma_{o, y}\right) & \text { if } x \in D^{-},\end{cases}
$$

where $\omega$ is the harmonic measure, $D^{+}=\Phi_{o, y}\left(\left\{\left(x_{1}, x_{2}\right) \in B: x_{2}>0\right\}\right)$, and $D^{-}=\Phi_{o, y}\left(\left\{\left(x_{1}, x_{2}\right) \in B: x_{2}<0\right\}\right)$.

When $D$ is the strip $\{z:-\pi / 2<\operatorname{Im} z<\pi / 2\}$ and $\Phi_{o, y}[(-1,1)]=(-\infty, \infty)$, explicit calculations (see [5]) show that

$$
e^{-2 d_{D}\left(z, \Gamma_{o}^{y}\right)}=\frac{(\cos \operatorname{Im} z)^{2}}{(1+|\sin \operatorname{Im} z|)^{2}}
$$

for all $z$ in the strip. On the other hand, when mapping the strip to the upper halfplane, a direct computation shows that

$$
\left(u_{o, y}(z)\right)^{2}=\left(1-\frac{2}{\pi}|\operatorname{Im} z|\right)^{2}
$$

for all $z$ in the strip. Therefore, by the conformal invariance of the hyperbolic distance and the harmonic measure,

$$
\left(u_{o, y}(x)\right)^{2} \approx e^{-2 d_{D}\left(x, \Gamma_{o}^{y}\right)}
$$

for all $x$ in $D$. Another property of the sets $\Omega_{i}$ (Theorem 5 in [13]) is that there exists an absolute constant $c$ with

$$
\int_{\Omega_{i}}\left(u_{o, y}(x)\right)^{2} d x \leq c \operatorname{diam} Q_{i} \operatorname{diam} q_{i},
$$

where $q_{i}$ is the largest Whitney cube in $\Omega_{i}$.

Consider $\gamma_{o}^{x_{i}}$, where $x_{i} \in q_{i}$. Following the argument in [18], we obtain

$$
\operatorname{diam} q_{i} \leq d\left(q_{i}, D^{c}\right) \leq d\left(x_{i}\right) \leq \frac{1}{C_{\Gamma}} d\left(\gamma_{o}^{x_{i}}, D^{c}\right) .
$$


Hence, for $y_{i} \in \gamma_{o}^{x_{i}} \cap \partial \Omega_{i}$, we have that $y_{i} \in I_{i} \cup I_{i+1}$ and

$$
\begin{aligned}
\operatorname{diam} q_{i} & \leq c d\left(y_{i}\right) \\
& \leq c\left(\text { length }\left(I_{i}\right)+\operatorname{length}\left(I_{i+1}\right)\right) \\
& \leq c\left(\operatorname{diam} Q_{i}+\operatorname{diam} Q_{i+1}\right) .
\end{aligned}
$$

We conclude that

$$
\int_{\Omega_{i}} e^{-2 d_{D}\left(x, \Gamma_{o}^{y}\right)} d x \leq c\left(\operatorname{diam} Q_{i+1}+\operatorname{diam} Q_{i}\right) \operatorname{diam} Q_{i} .
$$

Thus

$$
\int_{\bigcup_{i \in Z \backslash\{0\}} \Omega_{i} \cap A_{1}} e^{-2 d_{D}\left(x, \Gamma_{o}^{y}\right)} d x \leq c A\left(\frac{\sqrt{2}}{C_{\Gamma} 2^{k_{0}}}, D\right) \leq c \eta .
$$

Now we deal with the integral over $A_{1} \backslash \bigcup_{i \in Z \backslash\{0\}} \Omega_{i}$. If $z_{1} \in Q_{1} \cap \Phi_{o, y}[(0,1)] \cap$ $I_{1}$, then $z_{1}=\Phi_{o, y}(r)$ for some $r \in(0,1)$. Following [16, Sec. 10.3] and using the conformal invariance of the hyperbolic metric, we can suppose that $\Phi_{o, y}[(r, 1)]$ is $(0,1)$ and that $I_{1}$ is the union of two rays from 0 to the part of the boundary of $B$ contained in $\left\{\left(x_{1}, x_{2}\right): x_{1}<0\right\}$. Then, for all $z \in I_{1}$,

$$
d_{D}\left(z, \Phi_{o, y}[(r, 1)]\right)=d_{D}\left(z, z_{1}\right)
$$

From this identity, the corresponding one for $I_{-1}$, and the fact that the piece of the geodesic that goes from $Q_{-1}$ to $Q_{1}$ is contained in $D_{C_{\Gamma} / 2^{k}}$, one sees that there exists a $k_{\beta}$, depending only on $\beta$ and $k_{0}$, such that

$$
\lim _{\beta \rightarrow 0} k_{\beta}=\infty \quad \text { and } \quad d_{D}\left(z, \Gamma_{o}^{y}\right) \geq k_{\beta} \quad \text { for all } z \in A_{1} \backslash \bigcup_{i \in Z \backslash 0} \Omega_{i} .
$$

Hence we see that, for every $y \in D$,

$$
\lim _{\beta \rightarrow 0} \int_{z \in A_{1} \backslash \bigcup_{i \in Z \backslash 0} \Omega_{i}} e^{-2 d_{D}\left(x, \Gamma_{o}^{y}\right)} d x=0 .
$$

It remains to prove that the convergence is uniform in $y$. Since $\lim _{x \rightarrow \infty} d(x)=$ 0 , there is a finite number of Whitney cubes of diameter $\sqrt{2} / 2^{k_{0}}$, say $Q^{1}, \ldots, Q^{r}$. Let $Q$ be a Whitney cube and take $w, y$ in $Q$. Another property of the hyperbolic metric is that the distance from any side of a geodesic triangle to the other two sides is at most four. Hence there exists an absolute constant $K_{2}$ such that

$$
d_{D}\left(z, \gamma_{o}^{y}\right) \leq K_{2}
$$

for all $z \in \gamma_{o}^{w}$. Thus there exist $z_{1}, \ldots, z_{r}$ with

$$
d_{D}\left(z, \gamma_{o}^{w}\right)+K_{2} \geq \min _{i \in\{1, \ldots, r\}} d_{D}\left(z, \gamma_{o}^{z_{i}}\right)
$$

for all $z \in D$ and for all $w \in \bigcup_{i=1}^{r} Q^{i}$. Then, for all $x \in D$,

$$
e^{-2 d_{D}\left(x, \Gamma_{o}^{y}\right)} \leq c \max _{1 \leq i \leq r} e^{-2 d_{D}\left(x, \Gamma_{o}^{z_{i}}\right)} .
$$

From (26) and (27), 


$$
\lim _{\beta \rightarrow 0} \int_{Z \in A_{1} \backslash \bigcup_{i \in Z \backslash 0} \Omega_{i}} e^{-2 d_{D}\left(x, \Gamma_{o}^{y}\right)} d x=0
$$

uniformly in $y$. By (25) and the last formula, we conclude that $\lim _{\beta \rightarrow 0} I_{A_{1}}=0$ uniformly in $y$ and $z$.

It is left to show that

$$
\lim _{\beta \rightarrow 0} \int_{A_{2}} \frac{G_{D}(y, x) G_{D}(x, z)}{G_{D}(z, y)} d x=0
$$

uniformly in $y$ and $z$. Toward this end, consider

$$
\begin{aligned}
& A_{2}^{1}=\left\{x \in D \backslash F_{\beta}: d_{D}(x, y) \geq 1, d_{D}(x, z) \leq 1\right\}, \\
& A_{2}^{2}=\left\{x \in D \backslash F_{\beta}: d_{D}(x, y) \leq 1, d_{D}(x, z) \geq 1\right\}, \\
& A_{2}^{3}=\left\{x \in D \backslash F_{\beta}: d_{D}(x, y) \leq 1, d_{D}(x, z) \leq 1\right\} .
\end{aligned}
$$

If $A_{2}^{1} \neq \varnothing$ then the equivalence of the hyperbolic and quasihyperbolic distance implies the existence of $k_{1}, k_{2}$ with

$$
d(z) \leq \beta k_{1} \quad \text { and } \quad\left\{x \in D: d_{D}(x, z) \leq 1\right\} \subset k_{2} Q_{z},
$$

where $Q_{z}$ is a Whitney cube that contains $z$. Hence

$$
\begin{aligned}
\int_{A_{2}^{1}} \frac{G_{D}(y, x) G_{D}(x, z)}{G_{D}(z, y)} d x & \leq \int_{A_{2}^{1}} e^{2\left(d_{D}(y, z)-d_{D}(x, y)\right)} G_{D}(x, z) d x \\
& \leq e^{2} \int_{k_{2} Q_{z}} G_{D}(x, z) d x .
\end{aligned}
$$

By (11) and the definition of the quasihyperbolic distance, we have that

$$
d_{D}(x, z) \geq \frac{1}{4} \rho_{D}(x, z) \geq \frac{|x-z|}{4 R_{D}},
$$

where $R_{D}$ is the radius of the largest ball contained in $D$. Thus

$$
\begin{aligned}
\int_{A_{2}^{1}} \frac{G_{D}(x, y) G_{D}(z, x)}{G_{D}(z, y)} d x & \leq e^{2} \int_{k_{2} Q_{z}} \log \left(\operatorname{coth} \frac{|x-z|}{4 R_{D}}\right) d x \\
& \leq c \int_{0}^{\beta C} r \log (\operatorname{coth} r) d r .
\end{aligned}
$$

In the same way, we can prove (29) if we replace $A_{2}^{1}$ by $A_{2}^{2}$. Moreover,

$$
\begin{aligned}
\int_{A_{2}^{3}} \frac{G_{D}(x, y) G_{D}(z, x)}{G_{D}(z, y)} d x & \leq c \int_{A_{2}^{3}} e^{2 d_{D}(y, z)}\left[\left(G_{D}(x, z)\right)^{2}+\left(G_{D}(x, y)\right)^{2}\right] d x \\
& \leq 2 c \int_{0}^{\beta C} r(\log (\operatorname{coth} r))^{2} d r .
\end{aligned}
$$

A simple application of L'Hôpital's rule shows that $r(\log (\operatorname{coth} r))^{n}$ remains bounded as $r \rightarrow 0$ for any positive integer $n$. Thus, (28) is true and the lemma is proved. 
Clearly Lemma 5 implies (20) and then Theorem 2 is proved. The proof of Corollary 1 follows exactly the arguments in [6, pp. 197, 198].

\section{Proof of Theorem 3}

Let us suppose that $\tilde{A}(D)<\infty$. Set $h(x)=G_{D}(o, x)$, and define

$$
\begin{aligned}
& U_{k}=\left\{x \in D: a^{k} \leq h(x) \leq a^{k+1}\right\}, \\
& \hat{U}_{k}=\left\{x \in D: h(x) \leq a^{k+1}\right\},
\end{aligned}
$$

where $a$ is the constant given by the Harnack inequality in (31). To prove Theorem 3 we use the following lemma, which follows from the arguments in the proofs of Lemma 5.1 and Theorem 1.2 in [8].

Lemma 6 [8]. Let D be a uniformly regular domain in $\mathbb{R}^{n}$ for which

$$
\sum_{-\infty}^{\infty} \sup _{x \in U_{k}} E^{x}\left(\tau_{U_{k}}\right)<\infty \text { and } \sum_{-\infty}^{-2} \sup _{x \in \hat{U}_{k}} E^{x}\left(\tau_{\hat{U}_{k}}\right)<\infty .
$$

Then $D$ is $I U$.

REMARK 2. It is important at this point to make some remarks concerning (30). It is well know that, for any planar domain $D$ of finite area, there exists a universal constant $c$ such that, for all $x$ in $D$,

$$
E^{x}\left(\tau_{D}\right) \leq c|D| .
$$

Since $U_{k} \cap U_{m}=\emptyset$ for $|k-m|>1$ and $\bigcup_{k} U_{k}=D$, we have that

$$
\sum_{-\infty}^{\infty} \sup _{x \in U_{k}} E^{x}\left(\tau_{U_{k}}\right) \leq \sum_{-\infty}^{\infty} c\left|U_{k}\right| \leq 2 c|D|<\infty
$$

for such domains. On the other hand, Bañuelos and Davis [6] give an example of a domain $D$ of finite area which is not IU. Therefore we cannot replace (30) simply by

$$
\sum_{-\infty}^{\infty} \sup _{x \in U_{k}} E^{x}\left(\tau_{U_{k}}\right)<\infty
$$

in Lemma 6. (In fact, this last sum implies the finiteness of the conditional lifetime; see [3].)

We shall now prove that Lemma 6 holds for tube domains under our assumptions. By [1, Thm. 2], any simply connected domain is uniformly regular. Therefore it is enough to prove (30).

Let $x \in D$ and $r \in N$, and suppose that $d(x) \leq 1 / 2^{r+2}$. Since any simply connected domain is uniformly regular, there exists a $C_{0}$ independent of $x, r, k$ with

$$
P^{x}\left(\tau_{\hat{U}_{k}}<\tau_{B\left(x, 1 / 2^{r}\right)}\right)>P^{x}\left(\tau_{D}<\tau_{B\left(x, 1 / 2^{r}\right)}\right)>C_{0}
$$


(see [8, Lemma 2.6(iii)]). On the other hand, suppose that $d(x) \geq 1 / 2^{r+2}$. Let $Q_{o}$ be the Whitney cube containing $o$, and set

$$
M=\left\{Q \in \mathcal{F}: Q \cap Q_{o} \neq \emptyset\right\} .
$$

Then there exist $c, C$ with $c \leq h(x) \leq C$ for all $x \in \partial M$. By the Harnack inequality there exists an $a>0$ such that

$$
a^{-\tilde{\rho}_{D}(o, x)-k^{\prime}} \leq c a^{-\tilde{\rho}_{D}(o, x)} \leq h(x) \leq C a^{\tilde{\rho}_{D}(o, x)} \leq a^{\tilde{\rho}_{D}(o, x)+k^{\prime}}
$$

for some $k^{\prime} \in N$. Hence, $k>\tilde{\rho}_{D}(0, x)+k^{\prime}$ implies that $x \notin U_{k}$. That is,

$$
P^{x}\left(\tau_{U_{k}}<\tau_{B\left(x, 1 / 2^{r}\right)}\right)=1 .
$$

If $k+1<-\tilde{\rho}_{D}(0, x)-k^{\prime}$, then $x \notin \hat{U}_{k}$ and again

$$
P^{x}\left(\tau_{U_{k}}<\tau_{B\left(x, 1 / 2^{r}\right)}\right)=P^{x}\left(\tau_{\hat{U}_{k}}<\tau_{B\left(x, 1 / 2^{r}\right)}\right)=1 .
$$

We conclude that, for all $x$ in $D$ :

(i) $P^{x}\left(\tau_{U_{k}}<\tau_{B\left(x, 1 / 2^{r}\right)}\right)>C_{0}$ for all integer $k$ with $|k|>\rho^{*}(r+2)+k^{\prime}$;

(ii) $P^{x}\left(\tau_{\hat{U}_{k}}<\tau_{B\left(x, 1 / 2^{r}\right)}\right)>C_{0}$ for all $k<-2$ with $k+1<-\rho^{*}(r+2)-k^{\prime}$.

Using [8, Lemma 3.3], we obtain a constant $C_{1}$ such that, for all $x$ in $D$,

$$
E^{x}\left(\tau_{\hat{U}_{k}}\right) \leq C_{1} \frac{1}{2^{2 r}} .
$$

Thus to prove (30) it is enough to prove

$$
\sum_{r=0}^{\infty} \frac{2}{2^{2 r}}\left(\rho^{*}(r+3)-\rho^{*}(r+2)+2 k^{\prime}\right)<\infty
$$

or (equivalently)

$$
\sum_{r=2}^{\infty} \frac{1}{2^{2 r}}\left(\rho^{*}(r+1)-\rho^{*}(r)\right)<\infty .
$$

Indeed, we shall prove

$$
\sum_{r=2}^{\infty} \frac{1}{2^{2 r}} \rho^{*}(r)<\infty
$$

By our hypothesis,

$$
\sum_{r=2}^{\infty} \frac{1}{2^{2 r}} \rho^{*}(r) \leq \sum_{r=2}^{\infty} \frac{1}{2^{2 r}}\left(\rho_{\tilde{\gamma}}\left(r+n_{D}\right)+m_{D}\right) .
$$

Let $n_{k}$ be the number of Whitney cubes of diameter $\sqrt{2} / 2^{k}$ that intersect $\tilde{\gamma}$. Take $Q_{k} \in \mathcal{F}$ with diam $Q_{k}=\sqrt{2} / 2^{k}$ and $Q_{k} \cap \tilde{\gamma} \neq \emptyset$. Let $\tilde{\gamma}_{k}$ be the piece of the geodesic from $o$ to $Q_{k}$. By the definition of WA domains there exists an $m \in N$ such that, for every $w \in \tilde{\gamma}_{k}$,

Then

$$
d(w) \geq \frac{1}{2^{k+m}}
$$




$$
\begin{aligned}
\sum_{r=2}^{\infty} \frac{1}{2^{2 r}}\left(\rho_{\tilde{\gamma}}\left(r+n_{D}\right)+m_{D}\right) & \leq 2 m_{D}+\sum_{r=0}^{\infty} \frac{1}{2^{2 r}} \sum_{i=0}^{r+n_{D}+m} n_{i} \\
& =2 m_{D}+4^{n_{D}+m} \sum_{r=0}^{\infty} \sum_{i=0}^{r+n_{D}+m} \frac{n_{i}}{2^{2 i}} \frac{1}{2^{2\left(r+n_{D}+m\right)-2 i}} \\
& \leq c\left(m_{D}+\tilde{A}(D)\right) .
\end{aligned}
$$

We conclude that $\tilde{A}(D)<\infty$ implies (30), so $D$ is IU. The other direction follows from [18, Thm. 1].

Theorem 3 says that any tube along geodesics with finite area is IU. As mentioned before, if $D$ is a WA domain with $\tilde{A}(D)<\infty$ then it is easy to prove that $D$ is a countable union of tubes along geodesics. If we also suppose that $\tilde{A}(D, \varepsilon) \rightarrow 0$ as $\varepsilon \rightarrow 0$ then the series in (33) converges uniformly, although (32) is no longer true. This leads us to believe that it is possible to prove Conjecture 1 with the methods used in this paper.

\section{Log Sobolev Inequalities and Tubes}

So far we have used probabilistic techniques to attack Conjecture 1. However, there is a well-known analytic method to prove IU based on logarithmic Sobolev inequalities.

THEOREM 7 [10]. Let $e^{-t A}$ be a symmetric Markovian semigroup on $L^{2}(X, d x)$, where $d x$ is a Borel measure on the locally compact second countable Hausdorff space X. Let $Q$ be the associated Dirichlet form with domain $\operatorname{Quad}(H)$. Suppose there are two continuous functions $\varepsilon(p)>0$ and $\Gamma(p)$ defined for $2<p<\infty$ such that

$$
\int_{D} f^{p} \log f d x \leq \varepsilon(p)\left\langle A f, f^{p-1}\right\rangle+\Gamma(p)\|f\|_{p}+\|f\|_{p}^{p} \log \|f\|_{p}
$$

for all $2<p<\infty$ and $f \in \bigcup_{t>0} e^{-t A}\left(L^{1} \cap L^{\infty}\right)_{+}$. If

$$
t=\int_{2}^{\infty} \frac{\varepsilon(p)}{p} d p<\infty \text { and } M=\int_{2}^{\infty} \frac{\Gamma(p)}{p} d p<\infty,
$$

then $e^{-t A}: L^{2} \rightarrow L^{\infty}$ and

$$
\left\|e^{-t A}\right\|_{\infty, 2} \leq e^{M}
$$

We will now apply this theorem to prove Theorem 4. To simplify the arguments we suppose that, for all $x \in \tilde{\gamma}$,

$$
d(x) \leq d(z)
$$

for all $z \in \tilde{\gamma}_{o}^{x}$. If $Q_{0} \rightarrow Q_{1} \rightarrow \cdots \rightarrow Q_{n}$ is a Whitney chain from $o$ to $x$, (36) says that $\operatorname{diam} Q_{i} \geq \operatorname{diam} Q_{i+1}$ for $0 \leq i \leq n-1$. 
Define

$$
\eta_{1}(k)=\frac{1}{2^{2 k}} \sum_{0}^{k} n_{i}
$$

Note that, by (10),

$$
\eta_{1}(k+1)-\eta_{1}(k)=\frac{1}{2^{2 k+2}}\left[n_{k+1}-3\left(n_{1}+\cdots+n_{k}\right)\right] \leq 0,
$$

and therefore $\eta_{1}$ is nonincreasing for sufficiently large $k$. Let $x \in D$ and $Q_{x} \in \mathcal{F}$ with $x \in Q_{x}$ and diam $Q_{x}=\sqrt{2} / 2^{n_{x}}$. Using (36), we obtain

$$
\begin{aligned}
d^{2}(x) \tilde{\rho}_{D}(o, x) & \leq d^{2}(x)\left(m_{D}+\sum_{0}^{n_{x}+n_{D}} n_{i}\right) \\
& \leq m_{D} d^{2}(x)+c \eta_{1}\left(n_{x}+n_{D}\right) .
\end{aligned}
$$

Define

$$
\eta(d(x))=m_{D} d^{2}(x)+c \eta_{1}\left(n_{x}+n_{D}\right) .
$$

This is a nondecreasing function for $d(x)$ small enough, and by (16) we have

$$
\lim _{d(x) \rightarrow 0} \eta(d(x))=0 .
$$

In addition,

$$
\begin{aligned}
\int_{0}^{1} \frac{\eta(r)}{r} d r & \leq m_{D}+c \sum_{0}^{\infty} \int_{1 / 2^{i+1}}^{1 / 2^{i}} 2^{i+1} \eta_{1}\left(i+n_{D}\right) \\
& \leq m_{D}+c \sum_{0}^{\infty} \eta_{1}\left(i+n_{D}\right) \\
& \leq m_{D}+c \tilde{A}(D)
\end{aligned}
$$

Thus, if $\tilde{A}(D)<\infty$ then there exists a $C_{3}>0$ such that

$$
\rho_{D}(x, x) \leq C_{3}\left[1+\frac{\eta(d(x))}{d^{2}(x)}\right],
$$

with $\eta(r) \rightarrow 0$ as $r \rightarrow 0, \eta(r)$ nondecreasing for $r$ small enough, and

$$
\int_{0}^{1} \frac{\eta(r)}{r} d r<\infty
$$

Let $x \in D$. It follows from (2) that there exists a $y \in \Gamma_{o}^{x}$ such that

$$
d(y)<d(x) \text { and } \rho_{D}(o, y)>\rho_{D}(x, o) .
$$

Thus,

$$
\sup \left\{\rho_{D}(o, x): d(x) \geq \varepsilon\right\} \leq \sup \left\{\rho_{D}(o, x): d(x)=\varepsilon\right\} \leq C_{3}\left[1+\frac{\eta(\varepsilon)}{\varepsilon^{2}}\right] .
$$

On the other hand, if $\varepsilon$ is small enough then 


$$
\sup \left\{\rho_{D}(o, x): d(x) \leq \varepsilon\right\} \leq C_{3}\left[1+\frac{\eta(\varepsilon)}{d^{2}(x)}\right] .
$$

Hence, there exists a $C>0$ such that, for every $\varepsilon>0$,

$$
\rho_{D}(o, x) \leq C\left[1+\frac{\eta(\varepsilon)}{d^{2}(x)}+\frac{\eta(\varepsilon)}{\varepsilon^{2}}\right] .
$$

Let $\varphi_{o}^{V}$ be the first eigenfunction of $H=H_{0}+V$. By the Harnack inequality there exists a $c>0$ such that, for all $x \in D$,

$$
\rho_{D}(o, x) \geq-c \log \varphi_{0}^{V}(x) .
$$

Using the Hardy inequality and the imbedding properties of $|V|$ (see [2, p. 194]), one sees that (38) implies

$$
-\int_{D} f^{2} \log \varphi_{0}^{V}(x) d x \leq c \eta(\varepsilon)\langle A f, f\rangle+c\left(\frac{\eta(\varepsilon)}{\varepsilon^{2}}+1\right)\|f\|_{2}+\|f\|_{2}^{2} \log \|f\|_{2} .
$$

By Lemma 4.4.1 (Rosen's lemma) and Lemma 2.2.6 in [10], we obtain inequality (34) with $\varepsilon(p)=\eta(s / \sqrt{p})$ and

$$
\Gamma(p)=\frac{c}{p}\left(\frac{p}{s^{2}} \varepsilon(p)+1+\frac{s}{\sqrt{p}}-\frac{1}{2} \log \frac{s}{\sqrt{p}}\right) .
$$

It is easy to see that (37) implies (35) with $t \rightarrow 0$ as $s \rightarrow 0$. Our result now follows from Theorem 5 .

REMARK 3. Note that condition (10) was only used to prove that $\eta_{1}$ is nonincreasing for $k$ large enough. Suppose $D$ is just a tube along $\tilde{\gamma}$, and define

$$
\eta_{1}(k)=\sup _{k \leq r} \frac{1}{2^{2 r}} \sum_{0}^{r} n_{i} .
$$

This is a nonincreasing function that converges to zero. However, (37) is no longer true. To obtain (37) and then intrinsic ultracontractivity, one could replace (10) by other conditions, one of the simplest being

$$
\sum_{0}^{\infty} \frac{k n_{k}}{2^{2 k}}<\infty \text {. }
$$

ACKNOWLEDGMENT. I would like to thank Professor Rodrigo Bañuelos, my academic advisor, for his guidance on this paper, which is part of my Ph.D. thesis.

\section{References}

[1] A. Ancona, On strong barriers and an inequality of Hardy for domains in $R^{n}$, J. London Math. Soc. (2) 34 (1986), 274-290.

[2] R. Bañuelos, Intrinsic ultracontractivity and eigenvalue estimates for Schrödinger operators, J. Funct. Anal. 100 (1991), 181-206.

[3] - Lifetime and heat kernel estimates in non-smooth domains, IMA Vol. Math. Appl., 42, pp. 37-48, Springer, New York, 1992. 
[4] - Lifetime of conditioned Brownian motion and related estimates for heat kernels, eigenfunctions and eigenvalues in Euclidean domains, MSRI Lectures, 1998 (http://www.msri.org/publications/In/index.html).

[5] R. Bañuelos and T. Carroll, Conditioned Brownian motion and hyperbolic geodesics in simply connected domains, Michigan Math. J. 40 (1993), 321-331.

[6] R. Bañuelos and B. Davis, Heat kernel, eigenfunctions, and conditioned Brownian motion in planar domains, J. Funct. Anal. 84 (1989), 188-200.

[7] - A geometrical characterization of intrinsic ultracontractivity for planar domains with boundaries given by the graphs of functions, Indiana Univ. Math. J. 41 (1992), 885-913.

[8] R. F. Bass and K. Burdzy, Lifetimes of conditioned diffusions, Probab. Theory Related Fields 91 (1992), 405-443.

[9] R. Coornaert and A. Papadopolous, Symbolic dynamics and hyperbolic groups, Lecture Notes in Math., 1539, Springer-Verlag, Berlin, 1993.

[10] E. B. Davies, Heat kernels and spectral theory, Cambridge Univ. Press, Cambridge, 1989.

[11] E. B. Davies and B. Simon, Ultracontractivity and the heat kernel for Schrödinger operators and Dirichlet Laplacians, J. Funct. Anal. 59 (1984), 335-395.

[12] B. Davis, Conditioned Brownian motion in planar domains, Duke Math. J. 57 (1988), 397-421.

[13] P. S. Griffin, G. C. Verchota, and A. L. Vogel, Distortion of the area and conditioned Brownian motion, Probab. Theory Related Fields 96 (1993), 385-413.

[14] W. K. Hayman, Subharmonic functions, vol. 2, London Math. Soc. Monographs, 20, Academic Press, London, 1989.

[15] G. Martin, Quasiconformal and bi-Lipschitz homeomorphisms, uniform domains and the quasihyperbolic metric, Trans. Amer. Math. Soc. 292 (1985), 169-191.

[16] Ch. Pommerenke, Univalent functions, Vandenhoeck and Ruprecht, Göttingen, 1975.

[17] R. Smits, Conditional Brownian motion, spectral gaps and rates to equilibrium of diffusions, Ph.D. dissertation, Purdue University, 1996.

[18] - Necessary and sufficient conditions for finiteness of lifetimes, Potential Theory 5 (1996), 513-521.

[19] E. Stein, Singular integrals and differentiability properties of functions, Princeton Univ. Press, Princeton, NJ, 1970.

Department of Mathematics

Purdue University

West Lafayette, IN 47907

mendez@math.purdue.edu 